\title{
Estimates of higher order fractional derivatives at extreme points
}

\author{
Mohammed Al-Refai ${ }^{\mathrm{a}}$, D. Baleanu ${ }^{\mathrm{b}, \mathrm{c}, *}$ \\ ${ }^{a}$ Department of Mathematical Sciences, United Arab Emirates University, P. O. Box 15551, Al Ain, UAE. \\ ${ }^{b}$ Department of Mathematics and Computer Science, Cankaya University, 06530 Ankara, Turkey. \\ ${ }^{c}$ Institute of Space Sciences, Magurele-Bucharest, Romania.
}

Communicated by X.-J. Yang

\begin{abstract}
We extend the results concerning the fractional derivatives of a function at its extreme points to fractional derivatives of arbitrary order. We also give an estimate of the error and present two examples to illustrate the validity of the results. The presented results are valid for both Caputo and Riemann-Liouville fractional derivatives. (C)2017 All rights reserved.
\end{abstract}

Keywords: Extreme points, higher order fractional derivatives, Caputo derivative, Riemann-Liouville derivative. 2010 MSC: 26A33.

\section{Introduction}

Fractional calculus is developing fast in both theoretical and applied aspects $[2,8,9,12,14,15,19-$ 21]. The powerful aspects of this type of calculus can be seen when we apply it to extract some hidden aspects of the dynamics of complex systems. Generalizing some fundamental concepts from classical calculus to the fractional counterpart is not an easy task, but once it was done the researchers got some original results. We mention that recently, Al-Refai [4] has derived new estimates concerning the fractional derivative of a function at its extreme points. The estimates were derived for the Caputo and RiemannLiouville fractional derivatives with fractional derivatives $0<\delta_{1}<1$ and $1<\delta_{2}<2$. These results were used to develop new maximum principles and establish existence and uniqueness results for several types of fractional equations $[1,3,5-7,11,17]$, and to develop numerical schemes for certain fractional differential equations [16, 18, 22]. Also, the results concerning the Riemann-Liouville fractional derivative, were used and generalized for weaker conditions on $[6,18]$. In this paper, we extend these results to fractional derivatives of arbitrary order.

\subsection{Preliminaries}

We recall the definitions and main results concerning the Caputo and Riemann-Liouville fractional derivatives, see $[10,13]$.

\footnotetext{
*Corresponding author

Email addresses: m_alrefai@uaeu.ac.ae (Mohammed Al-Refai), dumitru@cankaya.edu.tr (D. Baleanu)
} 
Definition 1.1. Let $f(t) \in C[0, T], \delta \geqslant 0$, and $\Gamma$ denotes the Euler gamma function. The left RiemannLiouville fractional integral is defined by

$$
\left(I_{0+}^{\delta} f\right)(t)= \begin{cases}\frac{1}{\Gamma(\delta)} \int_{0}^{t}(t-s)^{\delta-1} f(s) d s, & \delta>0 \\ f(t), & \delta=0 .\end{cases}
$$

Definition 1.2. Let $f(t) \in C^{n}[0, T]$, the left Caputo fractional derivative is defined by

$$
\left({ }_{0} D_{C}^{\delta} f\right)(t)=\left(I_{0+}^{n-\delta} \frac{d^{n}}{d t^{n}} f\right)(t)= \begin{cases}\frac{1}{\Gamma(n-\delta)} \int_{0}^{t}(t-s)^{n-\delta-1} f^{(n)}(s) d s, & n-1<\delta<n \in Z^{+}, \\ f^{(n)}(t), & \delta=n \in Z^{+} .\end{cases}
$$

Definition 1.3. For $f(t) \in C^{\mathfrak{n}}[0, T]$, the left Riemann-Liouville fractional derivative is defined by

$$
\left({ }_{0} D_{R}^{\delta} f\right)(t)=\left(\frac{d^{n}}{d t^{n}} I_{0+}^{n-\delta} f\right)(t)= \begin{cases}\frac{1}{\Gamma(n-\delta)} \frac{d^{n}}{d t^{n}} \int_{0}^{t}(t-s)^{n-\delta-1} f(s) d s, & n-1<\delta<n \in Z^{+}, \\ f^{(n)}(t), & \delta=n \in Z^{+} .\end{cases}
$$

Caputo and Riemann-Liouville fractional derivatives are related by

$$
\left({ }_{0} D_{C}^{\delta} f\right)(t)={ }_{0} D_{R}^{\delta}\left(f-\sum_{k=0}^{n-1} \frac{t^{k}}{k !} f^{(k)}(0)\right)(t),
$$

where

$$
{ }_{0} D_{R}^{\delta} t^{k}=\frac{\Gamma(k+1)}{\Gamma(k-\delta+1)} t^{k-\delta} .
$$

We recall that when $f(0)=f^{\prime}(0)=\cdots=f^{(n-1)}(0)=0$, we have $\left({ }_{0} D_{C}^{\delta} f\right)(t)=\left({ }_{0} D_{R}^{\delta} f\right)(t)$. The rest of the paper is organized as follows. In Section 2 we present the main results concerning the fractional derivative of arbitrary order of a function at its extreme points. In Section 3 we present some examples to illustrate the validity of the new results. We close up with some concluding remarks in Section 4.

\section{The main results}

We generalize the main results obtained in [4] to the fractional derivatives of arbitrary order. We have the following theorem.

Theorem 2.1. Let $\mathrm{f}(\mathrm{t}) \in \mathrm{C}^{\mathfrak{n}}[0, \mathrm{~T}]$ attain its global minimum at $\mathrm{t}_{0} \in(0, \mathrm{~T}]$, then

$$
\begin{aligned}
\left({ }_{0} D_{C}^{\delta_{n}} f\right)\left(t_{0}\right)= & -\sum_{k=0}^{n-1} \frac{1}{\Gamma\left(k+1-\delta_{n}\right)} t_{0}^{k-\delta_{n}} h_{n-1}^{(k)}(0) \\
& +\frac{1}{\Gamma\left(-\delta_{n}\right)} \int_{0}^{t_{0}}\left(t_{0}-s\right)^{-\delta_{n}-1} h_{n-1}(s) d s, \quad n-1<\delta_{n}<n,
\end{aligned}
$$

where

$$
h_{n-1}(t)=f(t)-\sum_{k=0}^{n-1} \frac{f^{(k)}\left(t_{0}\right)\left(t-t_{0}\right)^{k}}{k !} .
$$

Proof. We apply the induction arguments to prove the result. The proof has been obtained in [4], for $0<\delta_{1}<1$. Assume that the result holds true for $n-1<\delta_{n}<n$, in the following we prove that it also holds true for $n<\delta_{n+1}<n+1$. Let $0<\alpha<1$ be such that $\delta_{n+1}=\alpha+n$, then $n-1<\delta_{n}=n-1+\alpha<n$, and using the induction hypothesis, we have

$$
\left({ }_{0} D_{C}^{n-1+\alpha} f\right)\left(t_{0}\right)=-\sum_{k=0}^{n-1} \frac{1}{\Gamma(k+2-n-\alpha)} t_{0}^{k+1-n-\alpha} h_{n-1}^{(k)}(0)+\frac{1}{\Gamma(1-n-\alpha)} \int_{0}^{t_{0}}\left(t_{0}-s\right)^{-n-\alpha} h_{n-1}(s) d s .
$$


Applying the above equation for $f^{\prime}(t)$ yields

$$
\begin{aligned}
\left({ }_{0} D_{C}^{n-1+\alpha} f^{\prime}\right)\left(t_{0}\right)= & -\sum_{k=0}^{n-1} \frac{1}{\Gamma(k+2-n-\alpha)} t_{0}^{k+1-n-\alpha} s_{n-1}^{(k)}(0) \\
& +\frac{1}{\Gamma(1-n-\alpha)} \int_{0}^{t_{0}}\left(t_{0}-s\right)^{-n-\alpha} s_{n-1}(s) d s
\end{aligned}
$$

where

$$
s_{n-1}(t)=f^{\prime}(t)-\sum_{k=0}^{n-1} \frac{f^{(k+1)}\left(t_{0}\right)\left(t-t_{0}\right)^{k}}{k !}
$$

Thus, we can show that

1. $h_{n}^{\prime}(t)=s_{n-1}(t)$, and

2. $h_{n}\left(t_{0}\right)=h_{n}^{\prime}\left(t_{0}\right)=h_{n}^{\prime \prime}\left(t_{0}\right)=\cdots=h_{n}^{(n)}\left(t_{0}\right)=0$.

Hence, $h_{n}(t)=\left(t_{0}-t\right)^{n+1} \mu_{n}(t)$ for some $\mu_{n}(t) \in C[0, T]$ and $h_{n}^{(k+1)}(0)=s_{n-1}^{(k)}(0)$. Integrating by parts (2.1) it yields,

$$
\int_{0}^{t_{0}}\left(t_{0}-s\right)^{-n-\alpha} s_{n-1}(s) d s=\left.\left(t_{0}-s\right)^{-n-\alpha} h_{n}(s)\right|_{0} ^{t_{0}}-(n+\alpha) \int_{0}^{t_{0}}\left(t_{0}-s\right)^{-n-\alpha-1} h_{n}(s) d s .
$$

We have

$$
\lim _{s \rightarrow t_{0}} \frac{h_{n}(s)}{\left(t_{0}-s\right)^{n+\alpha}}=\lim _{s \rightarrow t_{0}}\left(t_{0}-s\right)^{1-\alpha} \mu_{n}(t)=0, \quad 0<\alpha<1,
$$

and hence

$$
\begin{aligned}
\frac{1}{\Gamma(1-n-\alpha)} \int_{0}^{t_{0}}\left(t_{0}-s\right)^{-n-\alpha} s_{n-1}(s) d s \\
=-\frac{t_{0}^{-n-\alpha} h_{n}(0)}{\Gamma(1-n-\alpha)}-\frac{n+\alpha}{\Gamma(1-n-\alpha)} \int_{0}^{t_{0}}\left(t_{0}-s\right)^{-n-\alpha-1} h_{n}(s) d s \\
=-\frac{t_{0}^{-n-\alpha} h_{n}(0)}{\Gamma(1-n-\alpha)}+\frac{1}{\Gamma(-n-\alpha)} \int_{0}^{t_{0}}\left(t_{0}-s\right)^{-n-\alpha-1} h_{n}(s) d s .
\end{aligned}
$$

Thus,

$$
\begin{aligned}
\left.{ }_{0} D_{C}^{n-1+\alpha} f^{\prime}\right)\left(t_{0}\right)= & -\sum_{k=0}^{n-1} \frac{1}{\Gamma(k+2-n-\alpha)} t_{0}^{k+1-n-\alpha} h_{n}^{(k+1)}(0)-\frac{t_{0}^{-n-\alpha} h_{n}(0)}{\Gamma(1-n-\alpha)} \\
& +\frac{1}{\Gamma(-n-\alpha)} \int_{0}^{t_{0}}\left(t_{0}-s\right)^{-n-\alpha-1} h_{n}(s) d s \\
= & -\sum_{k=1}^{n} \frac{1}{\Gamma(k+1-n-\alpha)} t_{0}^{k-n-\alpha} h_{n}^{(k)}(0)-\frac{t_{0}^{-n-\alpha} h_{n}(0)}{\Gamma(1-n-\alpha)} \\
& +\frac{1}{\Gamma(-n-\alpha)} \int_{0}^{t_{0}}\left(t_{0}-s\right)^{-n-\alpha-1} h_{n}(s) d s \\
= & -\sum_{k=0}^{n} \frac{1}{\Gamma(k+1-n-\alpha)} t_{0}^{k-n-\alpha} h_{n}^{(k)}(0)+\frac{1}{\Gamma(-n-\alpha)} \int_{0}^{t_{0}}\left(t_{0}-s\right)^{-n-\alpha-1} h_{n}(s) d s \\
= & -\sum_{k=0}^{n} \frac{1}{\Gamma\left(k+1-\delta_{n+1}\right)} t_{0}^{k-\delta_{n+1}} h_{n}^{(k)}(0)+\frac{1}{\Gamma\left(-\delta_{n+1}\right)} \int_{0}^{t_{0}}\left(t_{0}-s\right)^{-\delta_{n+1}-1} h_{n}(s) d s .
\end{aligned}
$$

The last equation proves the result, since $\left({ }_{0} D_{C}^{n-1+\alpha} f^{\prime}\right)\left(t_{0}\right)=\left({ }_{0} D_{C}^{n+\alpha} f\right)\left(t_{0}\right)=\left({ }_{0} D_{C}^{\delta_{n}+1} f\right)\left(t_{0}\right)$. 
Remark 2.2. The integral in (2.2) exists because

$$
\left(t_{0}-s\right)^{-n-\alpha-1} h_{n}(s)=\left(t_{0}-s\right)^{-n-\alpha-1}\left(t_{0}-s\right)^{n+1} \mu_{n}(s)=\left(t_{0}-s\right)^{-\alpha} \mu_{n}(s)
$$

for some $\mu_{n} \in C[0, T]$. Since $0<\alpha<1$, then $\left(t_{0}-s\right)^{-\alpha}$ is integrable on $\left[0, t_{0}\right]$ and the result is obtained as $\mu_{n}$ is continuous.

Corollary 2.3. Let $\mathrm{f}(\mathrm{t}) \in \mathrm{C}^{\mathrm{n}}[0, \mathrm{~T}]$ attain its global minimum at $\mathrm{t}_{0} \in(0, \mathrm{~T}]$, and $\mathrm{n}-1<\delta_{\mathrm{n}}<\mathrm{n}$.

1. If $\mathrm{f}^{(\mathrm{n})}(\mathrm{t}) \geqslant 0$ on $\left[0, \mathrm{t}_{0}\right]$, then

$$
\left({ }_{0} D_{C}^{\delta_{n}} f\right)\left(t_{0}\right) \geqslant-\sum_{k=0}^{n-1} \frac{1}{\Gamma\left(k+1-\delta_{n}\right)} t_{0}^{k-\delta} h_{n-1}^{(k)}(0) .
$$

2. If $\mathrm{f}^{(\mathrm{n})}(\mathrm{t}) \leqslant 0$ on $\left[0, \mathrm{t}_{0}\right]$, then

$$
\left({ }_{0} D_{C}^{\delta_{n}} f\right)\left(t_{0}\right) \leqslant-\sum_{k=0}^{n-1} \frac{1}{\Gamma\left(k+1-\delta_{n}\right)} t_{0}^{k-\delta} h_{n-1}^{(k)}(0) .
$$

Proof. By the Taylor series expansion

$$
h_{n-1}(t)=f(t)-\sum_{k=0}^{n-1} \frac{f^{(k)}\left(t_{0}\right)\left(t-t_{0}\right)^{k}}{k !}=\frac{f^{(n)}\left(\xi_{n}(t)\right)\left(t-t_{0}\right)^{n}}{n !}
$$

for some $t<\xi_{n}(t)<t_{0}$, we have

$$
\begin{aligned}
r_{n} & =\frac{1}{\Gamma\left(-\delta_{n}\right)} \int_{0}^{t_{0}}\left(t_{0}-s\right)^{-\delta_{n}-1} h_{n-1}(s) d s=\frac{1}{\Gamma\left(-\delta_{n}\right)} \int_{0}^{t_{0}}\left(t_{0}-s\right)^{-\delta_{n}-1} \frac{f^{(n)}\left(\xi_{n}(s)\right)\left(s-t_{0}\right)^{n}}{n !} d s \\
& =\frac{(-1)^{n}}{n ! \Gamma\left(-\delta_{n}\right)} \int_{0}^{t_{0}}\left(t_{0}-s\right)^{n-\delta_{n}-1} f^{(n)}\left(\xi_{n}(s)\right) d s .
\end{aligned}
$$

Since $n-1<\delta_{n}<n$, and

$$
\Gamma\left(-\delta_{n}\right)=\left\{\begin{array}{l}
>0, \\
<0, \text { when } n \text { is even, } n \text { is odd, }
\end{array}\right.
$$

we have $\frac{(-1)^{\mathfrak{n}}}{\Gamma\left(-\delta_{\mathfrak{n}}\right)}>0$, and

$$
r_{n}=\left\{\begin{array}{l}
\geqslant 0, \text { when } f^{(n)}(t) \geqslant 0 \\
\leqslant 0, \text { when } f^{(n)}(t) \leqslant 0
\end{array}\right.
$$

which proves the result.

Corollary 2.4. Let $\mathrm{f}(\mathrm{t}) \in \mathrm{C}^{\mathrm{n}}[0, \mathrm{~T}]$ attain its global minimum at $\mathrm{t}_{0} \in(0, \mathrm{~T}]$, and $\left|\mathrm{f}^{(\mathrm{n})}(\mathrm{t})\right| \leqslant \mathrm{M}, \mathrm{t} \in[0, \mathrm{~T}]$, then

$$
\left({ }_{0} D_{C}^{\delta_{n}} f\right)\left(t_{0}\right)=-\sum_{k=0}^{n-1} \frac{1}{\Gamma\left(k+1-\delta_{n}\right)} t_{0}^{k-\delta_{n}} h_{n-1}^{(k)}(0)+r_{n}, \quad n-1<\delta_{n}<n,
$$

where

$$
\left|r_{n}\right| \leqslant \frac{M t_{0}^{n-\delta_{n}}}{n !\left(n-\delta_{n}\right)\left|\Gamma\left(-\delta_{n}\right)\right|}
$$

Proof. From (2.3) we have

$$
r_{n}=\frac{(-1)^{n}}{n ! \Gamma\left(-\delta_{n}\right)} \int_{0}^{t_{0}}\left(t_{0}-s\right)^{n-\delta_{n}-1} f^{(n)}\left(\xi_{n}(s)\right) d s
$$

and thus

$$
\left|r_{n}\right| \leqslant \frac{M}{n !\left|\Gamma\left(-\delta_{n}\right)\right|} \int_{0}^{t_{0}}\left(t_{0}-s\right)^{n-\delta_{n}-1} d s=\frac{M}{n !\left|\Gamma\left(-\delta_{n}\right)\right|} \frac{t_{0}^{n-\delta_{n}}}{n-\delta_{n}},
$$

which completes the proof. 
Remark 2.5. Similar results corresponding to the fractional derivatives at global maximum points are getting by utilizing the above mentioned results on $-f(t)$.

Below we show the analogous results for the Riemann-Liouville fractional derivative.

Theorem 2.6. Let $\mathrm{f}(\mathrm{t}) \in \mathrm{C}^{\mathrm{n}}[0, \mathrm{~T}]$ attain its global minimum at $\mathrm{t}_{0} \in(0, \mathrm{~T}]$, then for $\mathrm{n}-1<\delta_{\mathrm{n}}<\mathrm{n}$, we have

$$
\left({ }_{0} D_{R}^{\delta_{n}} f\right)\left(t_{0}\right)=-\sum_{k=0}^{n-1} \frac{1}{\Gamma\left(k+1-\delta_{n}\right)} t_{0}^{k-\delta_{n}}\left[h_{n-1}^{(k)}(0)-f^{(k)}(0)\right]+\frac{1}{\Gamma\left(-\delta_{n}\right)} \int_{0}^{t_{0}}\left(t_{0}-s\right)^{-\delta_{n}-1} h_{n-1}(s) d s,
$$

where

$$
h_{n-1}(t)=f(t)-\sum_{k=0}^{n-1} \frac{f^{(k)}\left(t_{0}\right)\left(t-t_{0}\right)^{k}}{k !}
$$

Proof. We have

$$
\begin{aligned}
\left({ }_{0} D_{C}^{\delta_{n}} f\right)(t) & \left.=\left({ }_{0} D_{R}^{\delta_{n}}\left(f-\sum_{k=0}^{n-1} \frac{t^{k}}{k !} f^{(k)}(0)\right)\right)(t)={ }_{0} D_{R}^{\delta_{n}} f\right)(t)-\sum_{k=0}^{n-1} \frac{\Gamma(k+1)}{\Gamma\left(k+1-\delta_{n}\right)} \frac{t^{k-\delta_{n}}}{k !} f^{(k)}(0) \\
& =\left({ }_{0} D_{R}^{\delta_{n}} f\right)(t)-\sum_{k=0}^{n-1} \frac{t^{k-\delta_{n}}}{\Gamma\left(k+1-\delta_{n}\right)} f^{(k)}(0) .
\end{aligned}
$$

Applying the result in (2.4) yields

$$
\begin{aligned}
\left({ }_{0} D_{R}^{\delta_{n}} f\right)\left(t_{0}\right)= & -\sum_{k=0}^{n-1} \frac{t_{0}^{k-\delta_{n}}}{\Gamma\left(k+1-\delta_{n}\right)} h_{n-1}^{(k)}(0)+\sum_{k=0}^{n-1} \frac{t_{0}^{k-\delta_{n}}}{\Gamma\left(k+1-\delta_{n}\right)} f^{(k)}(0) \\
& +\frac{1}{\Gamma\left(-\delta_{n}\right)} \int_{0}^{t_{0}}\left(t_{0}-s\right)^{-\delta_{n}-1} h_{n-1}(s) d s \\
= & -\sum_{k=0}^{n-1} \frac{t_{0}^{k-\delta_{n}}}{\Gamma\left(k+1-\delta_{n}\right)}\left[h_{n-1}^{(k)}(0)-f^{(k)}(0)\right]+\frac{1}{\Gamma\left(-\delta_{n}\right)} \int_{0}^{t_{0}}\left(t_{0}-s\right)^{-\delta_{n}-1} h_{n-1}(s) d s
\end{aligned}
$$

which proves the result.

Remark 2.7. The condition $f(t) \in C^{\mathfrak{n}}[0, T]$ could be so restrictive for the Riemann-Liouville fractional derivative. It is known that the fundamental solutions of certain homogenous fractional differential equations of Riemann-Liouville type, possesses a singularity at $t=0$. However, this condition has been replaced by a weaker ones for $0<\delta_{1}<1$ and $1<\delta_{2}<2$, see [6, 18]. The problem wether these weaker conditions are applicable for higher order fractional derivatives is left for a future work.

Corollary 2.8. Let $\mathrm{f}(\mathrm{t}) \in \mathrm{C}^{\mathrm{n}}[0, \mathrm{~T}]$ attain its global minimum at $\mathrm{t}_{0} \in(0, \mathrm{~T}]$, and $\mathrm{n}-1<\delta_{\mathrm{n}}<\mathrm{n}$.

1. If $f^{(n)}(t) \geqslant 0$ on $\left[0, t_{0}\right]$, then

$$
\left({ }_{0} D_{R}^{\delta_{n}} f\right)\left(t_{0}\right) \geqslant-\sum_{k=0}^{n-1} \frac{t_{0}^{k-\delta_{n}}}{\Gamma\left(k+1-\delta_{n}\right)}\left[h_{n-1}^{(k)}(0)-f^{(k)}(0)\right] .
$$

2. If $\mathrm{f}^{(\mathrm{n})}(\mathrm{t}) \leqslant 0$ on $\left[0, \mathrm{t}_{0}\right]$, then

$$
\left({ }_{0} D_{R}^{\delta_{n}} f\right)\left(t_{0}\right) \leqslant-\sum_{k=0}^{n-1} \frac{t_{0}^{k-\delta_{n}}}{\Gamma\left(k+1-\delta_{n}\right)}\left[h_{n-1}^{(k)}(0)-f^{(k)}(0)\right] .
$$

Proof. The proof of this theorem is similar to the one from Corollary 2.3.

Remark 2.9. The estimate of the error term in equation (2.5) is valid for the Riemann-Liouville fractional derivative. 


\section{Illustrative examples}

To illustrate the validity of our results, we present two examples for the Riemann-Liouville fractional derivative.

Example 3.1. Consider $f(t)=t^{3}+\cosh (t-1), t \in[0,1]$, and $2<\delta_{3}<3$. Since $f(t)$ is continuous on $[0,1]$ and $f(0.4) \leqslant f(0) \leqslant f(1)$, then $f$ attains a global minimum at $t_{0} \in(0,1)$. Since

$$
f^{(3)}(t)=6-\sinh (t-1) \geqslant 6-\sinh (-1) \geqslant 0, \quad t \in[0,1],
$$

then applying the result in Corollary 2.8, it yields

$$
\left({ }_{0} D_{R}^{\delta_{3}} f\right)\left(t_{0}\right) \geqslant-\sum_{k=0}^{2} \frac{t_{0}^{k-\delta_{3}}}{\Gamma\left(k+1-\delta_{3}\right)}\left[h_{2}^{(k)}(0)-f^{(k)}(0)\right],
$$

where

$$
h_{2}(t)=f(t)-\sum_{k=0}^{2} \frac{f^{(k)}\left(t_{0}\right)\left(t-t_{0}\right)^{k}}{k !}=f(t)-f\left(t_{0}\right)-\frac{1}{2} f^{\prime \prime}\left(t_{0}\right)\left(t-t_{0}\right)^{2} .
$$

By performing direct calculations one can verify that

$$
-\sum_{k=0}^{2} \frac{t_{0}^{k-\delta_{3}}}{\Gamma\left(k+1-\delta_{3}\right)}\left[h_{2}^{(k)}(0)-f^{(k)}(0)\right]=\frac{\delta_{3}-1}{\Gamma\left(3-\delta_{3}\right)} t_{0}^{-\delta_{3}}\left[\frac{\delta_{3}}{2} t_{0}^{2} f^{\prime \prime}\left(t_{0}\right)+\left(\delta_{3}-2\right) f\left(t_{0}\right)\right],
$$

and hence

$$
\left({ }_{0} D_{R}^{\delta_{3}} f\right)\left(t_{0}\right) \geqslant \frac{\delta_{3}-1}{\Gamma\left(3-\delta_{3}\right)} t_{0}^{-\delta_{3}}\left[\frac{\delta_{3}}{2} t_{0}^{2} f^{\prime \prime}\left(t_{0}\right)+\left(\delta_{3}-2\right) f\left(t_{0}\right)\right] .
$$

Since $f(t) \geqslant 0, t \in[0,1], f^{\prime \prime}\left(t_{0}\right) \geqslant 0$, and $2<\delta_{3}<3$, the right hand side of the above inequality is non-negative and thus

$$
\left({ }_{0} D_{R}^{\delta_{3}} f\right)\left(t_{0}\right) \geqslant 0, \text { for all } 2<\delta_{3}<3 .
$$

Example 3.2. Consider $f(t)=-\cosh (t), t \in[0,1]$, and $3<\delta_{4}<4$. Since $f^{(4)}(t)=-\cosh (t) \leqslant 0, t \in[0,1]$, then applying the result in Corollary 2.8 yields

$$
\left({ }_{0} D_{R}^{\delta_{4}} f\right)\left(t_{0}\right) \leqslant-\sum_{k=0}^{3} \frac{t_{0}^{k-\delta_{4}}}{\Gamma\left(k+1-\delta_{4}\right)}\left[h_{3}^{(k)}(0)-f^{(k)}(0)\right],
$$

where

$$
h_{3}(t)=f(t)-\sum_{k=0}^{3} \frac{f^{(k)}\left(t_{0}\right)\left(t-t_{0}\right)^{k}}{k !}=f(t)-f\left(t_{0}\right)-\frac{1}{2} f^{\prime \prime}\left(t_{0}\right)\left(t-t_{0}\right)^{2}-\frac{1}{3 !} f^{\prime \prime \prime}\left(t_{0}\right)\left(t-t_{0}\right)^{3} .
$$

By performing direct calculations one can verify that

$$
\left({ }_{0} D_{R}^{\delta_{4}} f\right)\left(t_{0}\right) \leqslant \frac{\delta_{4}-1}{\Gamma\left(4-\delta_{4}\right)} t_{0}^{-\delta_{4}}\left[\frac{\delta_{4}\left(\delta_{4}-2\right)}{6} t_{0}^{3} f^{\prime \prime \prime}\left(t_{0}\right)-\frac{\delta_{4}\left(\delta_{4}-3\right)}{2} t_{0}^{2} f^{\prime \prime}\left(t_{0}\right)-f\left(t_{0}\right)\left(\delta_{4}-3\right)\left(\delta_{4}-2\right)\right] .
$$

Since $f(t)=-\cosh (t)$ attains a global minimum at $t_{0}=1$, the last inequality leads to

$$
\left({ }_{0} D_{R}^{\delta_{4}} f\right)(1) \leqslant \frac{\delta_{4}-1}{\Gamma\left(4-\delta_{4}\right)}\left[-\frac{\delta_{4}\left(\delta_{4}-2\right)}{6} \sinh (1)+\frac{\left(\delta_{4}-3\right)\left(3 \delta_{4}-4\right)}{2} \cosh (1)\right], \quad 3<\delta_{4}<4 .
$$


We remark here that, if $\delta_{4} \rightarrow 3$, the last inequality leads to

$$
\left({ }_{0} D_{R}^{\delta_{4}} f\right)(1) \leqslant-\sinh (1)=f^{(3)}(1),
$$

while if $\delta_{4} \rightarrow 4$, the inequality leads to

$$
\left({ }_{0} \mathrm{D}_{\mathrm{R}}^{\delta_{4}} \mathrm{f}\right)(1) \leqslant 0
$$

Also, the error term in $(2.5)$ is

$$
\left|\mathrm{r}_{\mathrm{n}}\right| \leqslant \frac{\cosh (1)}{4 ! \Gamma\left(-\delta_{4}\right)\left(4-\delta_{4}\right)} \leqslant \cosh (1), \quad 3<\delta_{4}<4
$$

This error term increases with $\delta$. It approaches zero as $\delta$ approaches 3 , and it approaches $\cosh (1)$ as $\delta$ approaches 4 .

\section{Concluding remarks}

We have obtained estimates of fractional derivatives of a function at its extreme points. The results were obtained for the fractional derivatives of Caputo and Riemann-Liouvile types. The results were obtained in the functional space $\mathrm{C}^{\mathrm{n}}[0, \mathrm{~T}]$, and the problem wether these results are valid for a more wider spaces is left for a future work. We have presented some examples to illustrate the applicability of the new results which can also, be used to study higher order fractional differential equations.

\section{Acknowledgment}

The first author gratefully acknowledges the support of the UAE University under the grant COS/IRG$11 / 15$.

\section{References}

[1] A. B. Abdulla, M. Al-Refai, A. Al-Rawashdeh, On the existence and uniqueness of solutions for a class of non-linear fractional boundary value problems, J. King Saud Univ. Sci., 28 (2016), 103-110. 1

[2] R. P. Agarwal, M. Benchohra, S. Hamani, A survey on existing results for boundary value problems of nonlinear fractional differential equations and inclusions, Acta Appl. Math., 109 (2010), 973-1033. 1

[3] M. Al-Refai, Basic results on nonlinear eigenvalue problems with fractional order, Electron. J. Differential Equations, 2012 (2012), 12 pages. 1

[4] M. Al-Refai, On the fractional derivative at extreme points, Electron. J. Qual. Theory Differ. Equ., 2012 (2012), 5 pages. $1,2,2$

[5] M. Al-Refai, Yu. Luchko, Maximum principle for the fractional diffusion equations with the Riemann-Liouville fractional derivative and its applications, Fract. Calc. Appl. Anal., 17 (2014), 483-498. 1

[6] M. Al-Refai, Yu. Luchko, Maximum principle for the multi-term time-fractional diffusion equations with the RiemannLiouville fractional derivatives, Appl. Math. Comput., 257 (2015), 40-51. 1, 2.7

[7] M. Al-Refai, Yu. Luchko, Analysis of fractional diffusion equations of distributed order: Maximum principles and its applications, Analysis, 36 (2016), 123-133. 1

[8] D. Baleanu, K. Diethelm, E. Scalas, J. J. Trujillo, Fractional Calculus: Models and Numerical Methods, World Scientific, World Scientific Publishing, Hackensack, (2012). 1

[9] D. Baleanu, O. Mustafa, Asymptotic Integration and Stability for Differential Equations of Fractional Order, World Scientific Publishing, Hackensack, (2015). 1

[10] A. A. Kilbas, H. M. Srivastava, J. J. Trujillo, Theory and Applications of Fractional Differential Equations, Elsevier, Amsterdam, (2006). 1.1

[11] Y. Luchko, Fractional diffusion and wave propagation, Springer, 2014 (2014), 36 pages. 1

[12] J. T. Machado, V. Kiryakova, F. Mainardi, Recent history of fractional calculus, Commun. Nonlinear Sci. Numer. Simul., 16 (2011), 1140-1153. 1

[13] I. Podlubny, Fractional Differential Equations, Academic Press, San Diego, (1999). 1.1

[14] J. Sabatier, O. P. Agarwal, J. A. Tenreiro Machado, Advances in Fractional Calculus, Theoretical Developments and Applications in Physics and Engineering, Springer, Netherlands, (2007). 1 
[15] S. G. Samko, A. A. Kilbas, O. I. Marichev, Fractional Integrals and Derivatives, Theory and Applications, Gordon and Breach, Switzerland, (1993). 1

[16] M. Stynes, J. L. Gracia, A finite difference method for a two-point boundary value problem with Caputo fractional derivative, IMA Journal of Numerical Analysis, 35 (2014), 698-721. 1

[17] M. Syam, M. Al-Refai, Positive solutions and monotone iterative sequences for a class of higher order boundary value problems of fractional order, J. Fract. Calc. Appl., 4 (2013), 147-159. 1

[18] W. Xie, J. Xiao, Z. Luo, Existence of solutions for Riemann-Liouville fractional boundary value problem, Abstract and Applied Analysis, Abstr. Appl. Anal., 2014 (2014), 9 pages. 1, 2.7

[19] X.-J. Yang, Fractional derivatives of constant and variable orders applied to anomalous relaxation models in heat-transfer problems, Thermal Science, 2016 (2016), 13 pages. 1

[20] X. J. Yang, A new fractional operator of variablr order: application in the description of anomalous diffusion, Physica A: Statis. Mechanics Appl., 481 (2017), 276-283.

[21] X. J. Yang, F. Gao, H. M. Srivastava, New rheological models within local fractional derivative, Rom. Rep. Phys., 2017 (2017), 12 pages. 1

[22] H. Ye, F. Liu, V. Anh, I. Turner, Maximum principle and numerical method for the multi-term time-space Riesz-Caputo fractional differential equations, Appl. Math. Comput., 227 (2014), 531-540. 1 